See discussions, stats, and author profiles for this publication at: https://www.researchgate.net/publication/326558948

\title{
Microporous membranes comprising conjugated polymers with rigid backbones enable ultrafast organic-solvent nanofiltration
}

Article in Nature Chemistry · July 2018

DOI: 10.1038/541557-018-0093-9

\section{CITATIONS}

6

11 authors, including:

Bin Liang

National Center for Nanoscience and Technology

8 PUBLICATIONS 182 CITATIONS

SEE PROFILE

Xiao He

National Center for Nanoscience and Technology

6 PUBLICATIONS 88 CITATIONS

SEE PROFILE
Baoying Shen

Lehigh University

2 PUBLICATIONS 6 CITATIONS

SEE PROFILE

Zahid Ali Ghazi

National Center for Nanoscience and Technology

17 PUBLICATIONS 223 CITATIONS

SEE PROFILE

Some of the authors of this publication are also working on these related projects:

Project chiral interfaces and assembly View project

Project Monodisperse Hollow Spheres with Sandwich Heterostructured Shells as High-Performance Catalysts via an Extended SiO2 Template Method View project 


\title{
Microporous membranes comprising conjugated polymers with rigid backbones enable ultrafast organic-solvent nanofiltration
}

\author{
Bin Liang ${ }^{1,2}$, Hui Wang ${ }^{1,2}$, Xinghua Shi ${ }^{1}{ }^{1}$, Baoying Shen', Xiao He1, Zahid Ali Ghazi', Niaz Ali Khan', \\ Haksong Sin ${ }^{1}$, Abdul Muqsit Khattak, Lianshan Li ${ }^{1 \star}{ }^{1 \star}$ and Zhiyong Tang ${ }^{1 \star}$
}

Conventional technology for the purification of organic solvents requires massive energy consumption, and to reduce such expending calls for efficient filtration membranes capable of high retention of large molecular solutes and high permeance for solvents. Herein, we report a surface-initiated polymerization strategy through C-C coupling reactions for preparing conjugated microporous polymer (CMP) membranes. The backbone of the membranes consists of all-rigid conjugated systems and shows high resistance to organic solvents. We show that 42-nm-thick CMP membranes supported on polyacrylonitrile substrates provide excellent retention of solutes and broad-spectrum nanofiltration in both non-polar hexane and polar methanol, the permeance for which reaches 32 and $22 \mathrm{I} \mathrm{m}^{-2} \mathrm{~h}^{-1}$ bar $^{-1}$, respectively. Both experiments and simulations suggest that the performance of CMP membranes originates from substantially open and interconnected voids formed in the highly rigid networks.

S ince the late 1960s, membrane technology has been broadly utilized in purification and separation process thanks to its extremely low energy consumption, reduced operation time, environmental compatibility and good safety compared with traditional purification processes involved with distillation, evaporation or adsorption ${ }^{1}$. Currently, this efficient separation technique is largely limited to applications in aqueous solutions, and one of the most successful examples is sea water desalination using reverse osmosis membranes composed of a polyamide separating layer and a porous ultrafiltration support ${ }^{2,3}$. In comparison, organic-solvent nanofiltration (OSN) is still regarded as an emerging separation technology, with its first commercial success in the early $1990 \mathrm{~s}^{4,5}$. Unlike aqueous nanofiltration, which relies on steric and electrostatic interaction synergy ${ }^{6,7}$, OSN is solely based on the physical size exclusion effect and imposes stringent requirements that are hardly satisfied with current polymers; for instance, the membranes must possess pores below $2 \mathrm{~nm}$ to reject solutes of a molecular weight less than $1,000 \mathrm{~g} \mathrm{~mol}^{-1}$ and simultaneously have excellent stability and high permeance in a wide range of organic solvents with different polarities ${ }^{8-11}$.

To reduce the thickness of the separating layer is the most straightforward and effective method to achieve highly permeable membranes for $\mathrm{OSN}^{10}$. However, unavoidable generation of defects inside the extremely thin membranes would give rise to weak mechanical strength. An alternative strategy is to design new molecular structures of high microporosity, continuous channels, and good chemical and structural robustness by improving the structure and shape persistence ${ }^{12-19}$. As examples, by adopting a rigid ladder-like polymer chain consisting of contorted spirobisindane linkage and dioxane bridges, polymers of intrinsic microporosity (PIMs) are prepared with high free volume due to considerable prevention of close interchain packing ${ }^{20}$. These PIM membranes show a substantial rejection rate of $90 \%$ for hexaphenylbenzene, along with a simultaneous increased permeance of $181 \mathrm{~m}^{-2} \mathrm{~h}^{-1} \mathrm{bar}^{-1}$ for $n$-heptane. Nevertheless, an anomalous decrease in permeance is observed when the membrane thickness is less than $140 \mathrm{~nm}$ due to the structural relaxation of one-dimensional chains in PIMs. Further enhancement of the chain rigidity of PIMs has been achieved by replacing spirobisindane and dioxane units with more rigid ethanoanthracene (EA) and Troger's base (TB) to produce PIM-EA-TB ${ }^{13}$. Despite the much more improved chain rigidity, PIM-EA-TB still suffers from one-dimensional chain structure with considerable structural flexibility and solubility in organic solvent. Thereafter, three-dimensional (3D) cross-linked polyarylate microporous polymers were synthesized using the contorted monomers of spiro-structured 5,5',6,6'-tetrahydroxy-3,3,3',3'-tetramethylspirobisindane and cardo-structured 9,9-bis(4-hydroxyphenyl) fluorine, which exhibit decent separation performance in organic solvent ${ }^{21}$. It needs to be pointed out that utilization of contorted monomers enhances the structural rigidity and microporosity to some extent, but the structural persistence and chemical stability of these membranes are still not satisfactory due to the intrinsic flexibility and reactivity of the ester linkers.

Herein, we propose a novel type of OSN membrane based on all-rigid porous frameworks composed of only chemically inert carbon-carbon and carbon-hydrogen bonds. In sharp contrast with the most widely used polyamide or polyarylate materials without permanent pores, conjugated microporous polymers (CMPs) possess abundant, interconnected and permanent micropores in $3 \mathrm{D}$ networks consisting of all-rigid $\pi$-conjugated skeletons ${ }^{22}$. Furthermore, CMPs could offer tunable pore size and pore structure via molecular engineering of their conjugated skeletons ${ }^{23}$. Thus, when used as OSN membranes, CMPs are expected to effectively prevent structural relaxation in various solvents, while their microporous nature will afford large solute retention and high solvent permeance.

\section{Results and discussion}

Construction and characterization of CMP membranes. A big challenge for the fabrication of CMP membranes is the insolubility 
and poor processability of CMPs after formation via solution-phase polymerization. In this work, a surface-initiated polymerization strategy was developed for the preparation of large-area and defect-free CMP membranes for ultrafast OSN. The CMP membranes were first fabricated on bromobenzene-functionalized silica wafers via surface-initiated Sonogashira-Hagihara polymerization of 1,3,5-triethynylbenzene (1,3,5-TEB) with three different dibromobenzenes, 1,4-dibromobenzene, 1,3-dibromobenzene and 1,2-dibromobenzene. The corresponding highly cross-linked, allconjugated products were named $p$-CMP, $m$-CMP and $o$-CMP, respectively (Supplementary Fig. 1). Afterwards, the as-prepared CMP membranes were easily transferred to the top of porous supports to form a composite membrane (Supplementary Fig. 2). Macro and micro images of a typical $4 \mathrm{~cm} \times 4 \mathrm{~cm} p$-CMP membrane on a porous polyacrylonitrile (PAN) substrate show a uniform lightyellow membrane covering the porous PAN, with a pore size of tens to hundreds of nanometres (Fig. 1b and Supplementary Fig. 3). The contact angles of all $p$-CMP, $m$-CMP and $o$-CMP membranes were in the range of 100 to $120^{\circ}$, suggesting they have a hydrophobic nature (inset in Fig. 1b and Supplementary Fig. 4). The morphology and structure of the as-synthesized membranes were carefully investigated using various microscopy and spectroscopy techniques. Large-area scanning electron microscope (SEM) images showed that the membranes were flexible, freestanding and mechanically robust (Supplementary Fig. 5), while cross-section SEM images indicated that the thickness of CMP membranes was around $50 \mathrm{~nm}$ (inset in Fig. 1c). Accurate thicknesses of the membranes were obtained using the height profiles of atomic force microscope (AFM) images, and the values were about 42,45 and $44 \mathrm{~nm}$ for $p$-CMP, $m$-CMP and $o$-CMP, respectively (Fig. 1d,e and Supplementary Fig. 6). In addition, the surface roughness of the membranes was discerned from magnified SEM and AFM images (Supplementary Fig. 7). High-resolution transmission electron microscope images clearly manifested the amorphous nature of all the CMP membranes (Supplementary Fig. 8), which was further confirmed by their wideangle X-ray diffraction patterns (Supplementary Fig. 9). Finally, Fourier-transform infrared spectra of all the CMP membranes displayed characteristic $-\mathrm{C} \equiv \mathrm{C}$ - stretching peaks at $2,200 \mathrm{~cm}^{-1}$, revealing successful polymerization of 1,3,5-TEB and varied dibromobenzene monomers (Supplementary Fig. 10).

The all-conjugated structure constructed only with carboncarbon and carbon-hydrogen bonds endows CMP membranes much higher structural rigidity compared with typical OSN membranes. First, molecular modelling discloses that it is much more difficult to bend the alkynyl linkers in CMPs with respect to the amide and ester linkers in 3D polyamide and polyarylate, respectively, as well as the EA and TB linkers in newly developed PIMs (Fig. If and Supplementary Fig. 11). Second, wrinkling-based measurement ${ }^{10}$ under compressive stress induced by an elastomer substrate revealed that the Young's modulus of CMP membranes is in the range of 4-6 GPa, depending on the dibromobenzene linker used (Fig. 1g and Supplementary Figs. 12 and 13). In comparison, the traditional polyamide membrane showed a value of only $0.36 \mathrm{GPa}$. Finally, peak force quantitative nanomechanical mapping (PFQNM) also disclosed that all the CMP membranes possessed a high Young's modulus of 6-7 GPa, while the modulus of the polyamide membrane was only $0.57 \mathrm{GPa}$ (Fig. $1 \mathrm{~h}$ ). It should be noted that although absolute values obtained by wrinkling and PFQNM methods are slightly different, both methods verify that the Young's modulus of CMPs is one order of magnitude higher than that of the traditional polyamide. The Young's moduli of CMPs, polyamide and previously reported polyarylate and PIMs are summarized in Supplementary Table 1, demonstrating better structural rigidity of the CMP membranes. This high structural rigidity gives rise to a relatively higher surface area and permanent pores, which are crucial for ultrafast and stable nanofiltration. $\mathrm{N}_{2}$ adsorption isotherms at $77 \mathrm{~K}$ showed Brunauer-Emmett-Teller surface areas of 513, 383 and $593 \mathrm{~m}^{2} \mathrm{~g}^{-1}$ for $p$-CMP, $m$-CMP and $o$-CMP, respectively, while the pore sizes of $p$-CMP and $m$-CMP were below $1.5 \mathrm{~nm}$ (that is, well in the range of nanofiltration). Exceptionally, the largest pore size of $o$-CMP was around $2.2 \mathrm{~nm}$, indicating that the topology of monomers affects the pore size of products (Supplementary Fig. 14 and Supplementary Table 2).

OSN performances of CMP membranes. The OSN performances of CMP membranes were evaluated based on CMP/PAN composites (Fig. 1b and Supplementary Fig. 3). Evidently, $p$-CMP and $m$-CMP showed very similar rejection behaviour, with a clear molecule weight cut-off around $560 \mathrm{~g} \mathrm{~mol}^{-1}$ (Fig. 2a, Supplementary Fig. 15 and Supplementary Table 3), whereas all dye rejections were less than $90 \%$ for the $o$-CMP membrane. This result is consistent with the fact that $o$-CMP has much larger pores of $2.2 \mathrm{~nm}$ (Supplementary Fig. 14 and Supplementary Table 2). Figure 2b shows that protoporphyrin-IX (PPh-IX), with a molecular weight of $562.7 \mathrm{~g} \mathrm{~mol}^{-1}$ and a width of $1.47 \mathrm{~nm}$, was completely rejected by the $p$-CMP membrane. It is noteworthy that the molecular size of PPh-IX $(1.47 \mathrm{~nm})$ is almost the same as the size of the largest pore $(1.5 \mathrm{~nm})$ in $p$-CMP, implying that the rejection originated exclusively from pore-size exclusion.

The permeation properties of different organic solvents through the CMP membrane were systematically investigated. It was observed that the applied pressure during initial filtration could cause physical ageing and compaction of PAN supports, leading to a decrease of about $48.2 \%$ in permeance of CMP/PAN membranes over the first $8 \mathrm{~h}$ (Supplementary Fig. 16); therefore, all the permeances reported in this work were obtained after a steady flux state was reached. The permeance of different solvents at a pressure difference of 1 bar is plotted, along with their viscosities, in Fig. 2c. Notably, solvent permeance increased in inverse proportion to viscosity (Supplementary Fig. 17a-c and Supplementary Table 4), suggesting that there is no specific interaction between the hydrophobic pore and varied solvents ${ }^{24}$. Furthermore, the flux-corresponding to the permeance multiplied by the applied pressure-increased linearly with applied pressure (Supplementary Fig. 17d). Of note, the product of permeance and viscosity is a constant, independent of Hansen solubility parameters (Supplementary Fig. 18), which discloses that solvent transport in CMP membranes follows the poreflow mode ${ }^{25,26}$. This result validates the predication that pore flow could be obtained in membranes with permanent micropores ${ }^{27}$. Armed with these results, one can deduce that the permeance of solvents through CMP membranes fits well with the classic HagenPoiseuille equation:

$$
P=J / \Delta p=\varepsilon \pi \gamma_{\mathrm{p}}^{2} / 8 \mu \delta \tau
$$

where $P$ is the permeance, $J$ is the solvent flux, $\Delta p$ is the applied pressure, $\varepsilon$ is the surface porosity, $\gamma_{\mathrm{p}}$ is the pore radius, $\mu$ is the viscosity, $\delta$ is the membrane thickness and $\tau$ is the tortuosity. Impressively, in the case of the 42-nm-thick p-CMP membrane, hexane, with the smallest viscosity $(0.31 \mathrm{mPa} \mathrm{s})$, gave the highest permeance of $32 \mathrm{l} \mathrm{m}^{-2} \mathrm{~h}^{-1} \mathrm{bar}^{-1}$, followed by methanol $(0.49 \mathrm{mPa} \mathrm{s})$, with the second highest value of $22 \mathrm{l} \mathrm{m}^{-2} \mathrm{~h}^{-1}$ bar $^{-1}$ (black squares in Fig. 2c). Aromatic solvents of toluene and chlorobenzene showed low permeances of 4.2 and $1.41 \mathrm{~m}^{-2} \mathrm{~h}^{-1} \mathrm{bar}^{-1}$, respectively, despite their relatively low viscosity. This deviation has been discerned previously and generally ascribed to the bulky structure of the phenyl ring ${ }^{24}$. Nevertheless, the permeances of both methanol and hexane are even higher than the values of recently reported 8-nm-thick polyamide membranes supported on an XP84 support (menthol and heptane permeances are 19 and $\sim 11 \mathrm{~m}^{-2} \mathrm{~h}^{-1} \mathrm{bar}^{-1}$, respectively $)^{10}$. More importantly, in contrast with the 8 -nm-thick 
<smiles>CCOc1c(C)c(OCC)c(C)c(OCOC)c1C</smiles>

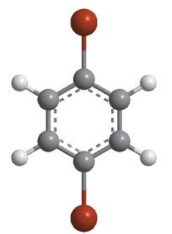<smiles>Cc1c(C)c(C)c([O-])c(C)c1C</smiles>
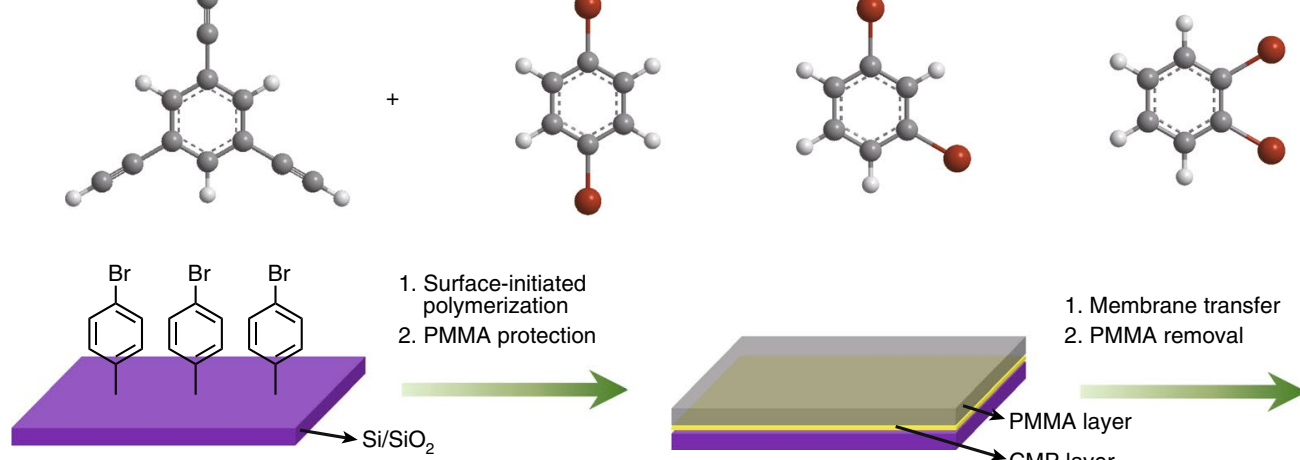

1. Surface-initiated polymerization

2. PMMA protection

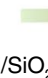

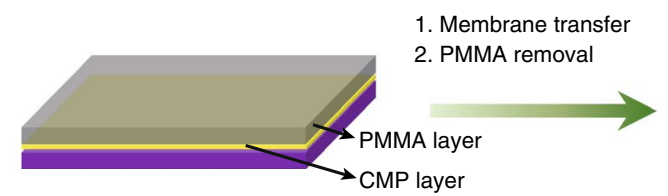

d

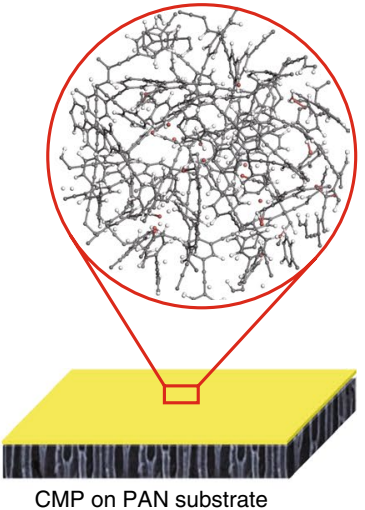

CMP on PAN substrate b

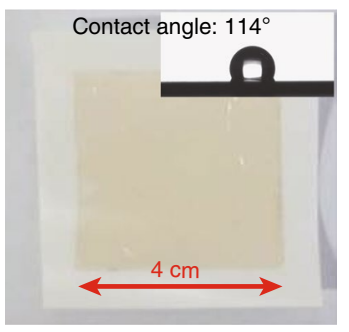

c

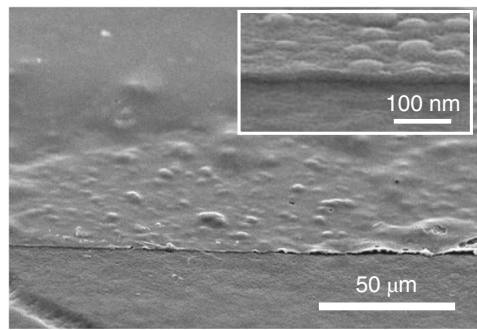

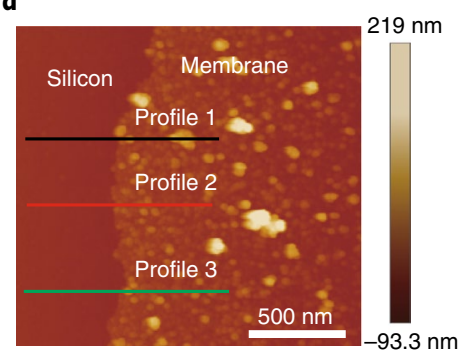

e

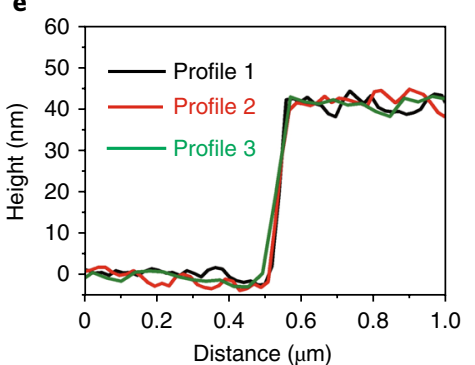

f

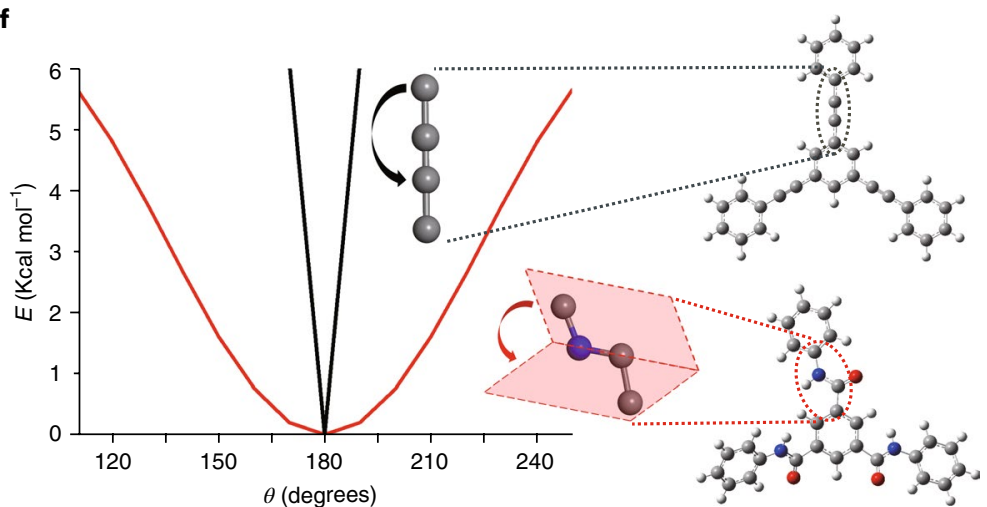

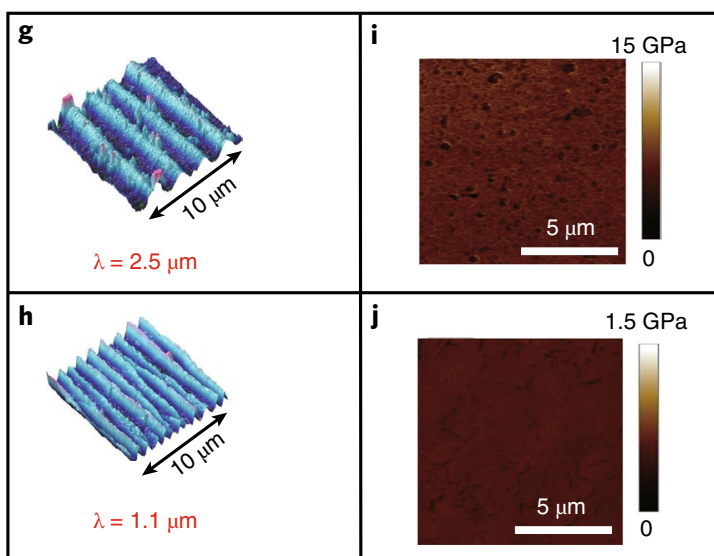

Fig. 1 | Preparation and characterization of all-conjugated CMP membranes with various dibromobenzene monomers, and investigation of their mechanical properties. a, Surface-initiated polymerization of the CMP membrane on a bromobenzene-functionalized $\mathrm{Si} / \mathrm{SiO}_{2} \mathrm{substrate}$. A SonogashiraHagihara reaction between 1,3,5-triethynylbenzene (1,3,5-TEB) and three different dibromobenzenes (whose structures are shown at the top) was adopted because it gives highly cross-linked and all-conjugated porous polymers; the bromine sites on the $\mathrm{Si} / \mathrm{SiO}_{2}$ substrate (bottom left) acted as the initial reaction sites. The dibromo-monomers were used: 1,4-dibromobenzene, 1,3-dibromobenzene and 1,2-dibromobenzene. Atoms colour code: $\mathrm{C}$, grey; $\mathrm{H}$, white; $\mathrm{Br}$, red. The bottom panel shows a schematic representation of the surface-initiated polymerization; the inset in the red circle (right) shows the all-rigid skeleton of the resulting CMP membrane. $\mathbf{b}$, Photograph of an approximately 42 -nm-thick $p$-CMP membrane transferred to a PAN substrate. The contact angle is $114^{\circ}$ (inset), indicating the hydrophobic nature of this membrane. c, Large-area surface and cross-section (inset) SEM images of the CMP membrane after transferring it to a silicon substrate. d,e, AFM image (d) and corresponding height profile (e) of a p-CMP membrane on top of a silicon wafer. A scratch was made to expose the wafer surface, allowing measurement of the height from the silicon wafer surface to the upper membrane surface. $\mathbf{f}$, Comparison of the chemical structure rigidity of CMPs and that of a conventional polyamide polymer. Molecular modelling confirmed that the alkyne linker (black curve) was much more rigid than the amide linker (red curve), with much less variation in the angles shown as insets, leading to the highly rigid backbone of the CMPs (atom colour key: $\mathrm{C}$, grey; $\mathrm{H}$, white; $\mathrm{N}$, blue; $\mathrm{O}$, red). $\mathbf{g}, \mathbf{h}, \mathrm{AFM}$ images of the wrinkles formed when the $p$ - $\mathrm{CMP}(\mathbf{g}$ ) or polyamide membrane $(\mathbf{h})$ was transferred onto an elastomer substrate and subjected to applied compressive stress. i,j, Young's moduli of $p$-CMP (i) and polyamide membrane $(\mathbf{j})$, tested using the PFQNM method.

polyamide membrane showing the lowest permeance of non-polar heptane, our CMP membranes possessed the highest permeance of non-polar solvent (for example, hexane). This filtration behaviour differentiates the CMP membrane greatly from the conventional polar polyamide membrane and makes it more favourable for the organic system.
Figure $2 \mathrm{~d}$ summarizes the methanol permeance versus the reciprocal thickness for different membranes, including CMPs, polyarylate ${ }^{21}$, polyamide ${ }^{1,10}$, PIMs $^{1}$ and metal-organic framework composite ${ }^{28}$. Note that a dashed line, the slope of which represents the solvent permeability (permeance per unit thickness), was intentionally added in Fig. $2 \mathrm{~d}$ to reveal the intrinsic capability of membranes towards solvent 

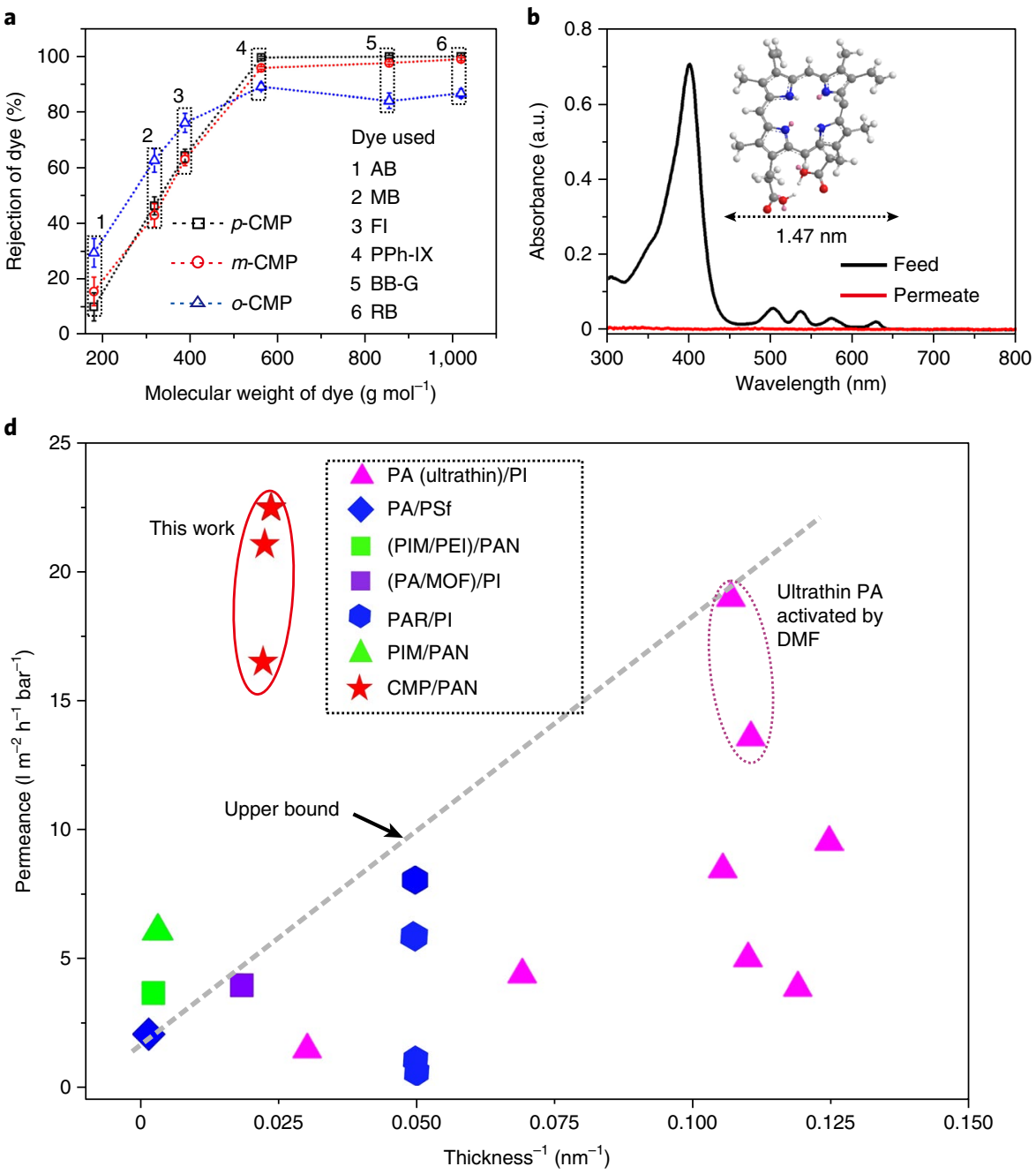
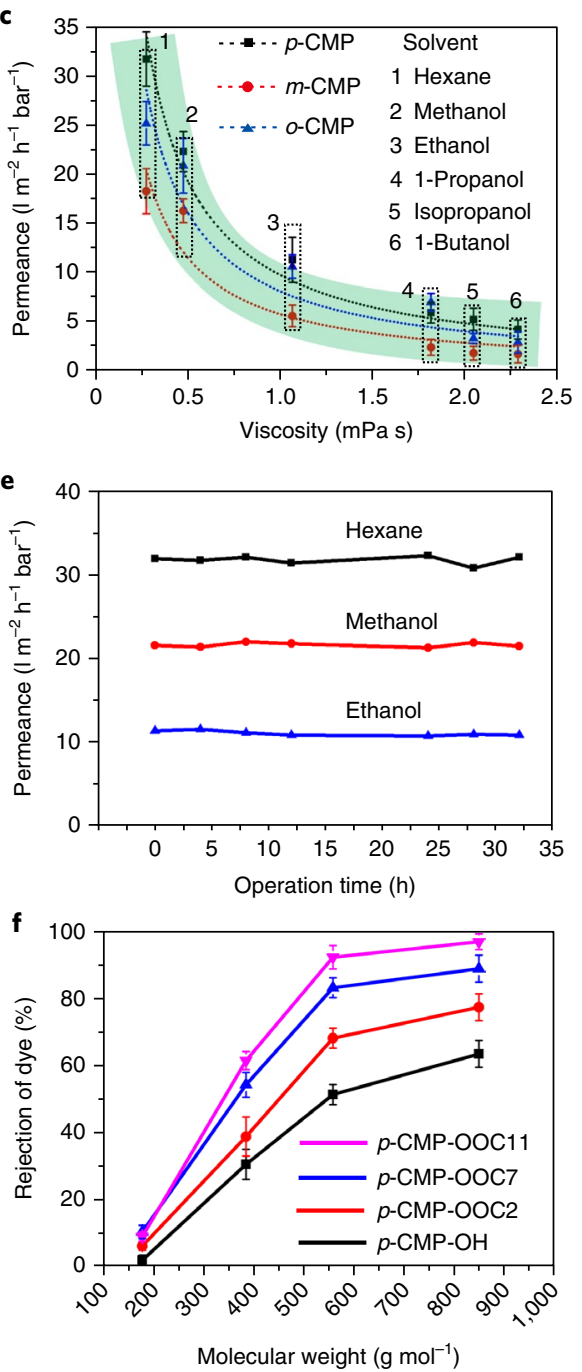

Fig. 2 | Nanofiltration performances of $p$-CMP, $\boldsymbol{m}$-CMP and o-CMP membranes. $\mathbf{a}$, Rejection behaviour of different CMP membranes versus the molecular weight of various dyes (rose bengal (RB; $\left.1,017 \mathrm{~g} \mathrm{~mol}^{-1}\right)$, brilliant blue G (BB-G; $\left.854 \mathrm{~g} \mathrm{~mol}^{-1}\right)$, PPh-IX (562 $\left.\mathrm{g} \mathrm{mol}^{-1}\right)$, fluorescein-4-isothiocyanate $\left(\mathrm{Fl} ; 389 \mathrm{~g} \mathrm{~mol}^{-1}\right)$, methylene blue $\left(\mathrm{MB} ; 320 \mathrm{~g} \mathrm{~mol}^{-1}\right)$ and azobenzene $\left(A B ; 188 \mathrm{~g} \mathrm{~mol}^{-1}\right)$ ) in ethanol. b, Ultraviolet visible absorption spectra of PPh-IX dye in methanol to evaluate the separation performance of the $p$-CMP membrane. Inset: molecular structure of PPh-IX ( $\mathrm{C}$, grey; $\mathrm{H}$, white; $\mathrm{N}$, blue; O, red; lonepair electrons, pink). c, Plot of solvent permeance through different CMP membranes against the solvent viscosity. The dashed curves show the reciprocal fitting of the permeance through $p$-CMP (black), $m$-CMP (red) and o-CMP (blue). The green shading shows an inverse relationship between permeance and viscosity. $\mathbf{d}$, Permeance for methanol versus the reciprocal membrane thickness for $p$-CMP, $m$-CMP and $o$-CMP. Previously reported polymer-based OSN membranes are included for comparison, that consist of polyamide (PA) ${ }^{1,10}, \mathrm{PIM}^{1}$, metal-organic framework (MOF) ${ }^{28}$ and polyarylate (PAR) ${ }^{21}$, $\mathrm{PI}$, polyimide; PSf, polysulfone; $\mathrm{PEI}$, polyetherimide; $\mathrm{DMF}, \mathrm{N}, \mathrm{N}$-dimethylformamide. The name of each membrane refers to its separating layer on the left and supporting substrate on the right. In two cases, the separating layer is a hybrid material: (PIM/PEI) and (PA/MOF). The dashed line shows the upper bond of the solvent permeability (permeance per unit thickness). e, Plot of hexane, methanol and ethanol permeances with time for $p$-CMP membranes. f, Rejection behaviour of post-modified $p-\mathrm{CMP}-\mathrm{OH}$ membranes by different moieties. All nanofiltration experiments were conducted in a dead-end cell at $25^{\circ} \mathrm{C}$ under 1 bar. The error bars in $\mathbf{a}, \mathbf{c}$ and $\mathbf{f}$ represent the standard deviation, which was calculated based on an average of three membranes.

transport. Interestingly, only two types of membrane with permanent pores (PIMs and CMPs) had permeance above the dashed line. From this result, it is reasonable to infer that solvent in dense polymer membranes goes through a rather low-efficiency solution-diffusion process, since there is a lack of permanent pores (samples below the dashed line). Unfortunately, the overall permeance of PIM membranes above the dashed line was quite low due to their poor structural persistence (green triangle and green square). In comparison, CMP membranes showed the best solvent permeance thanks to their high structural rigidity and abundant permanent micropores (red stars). In addition, a plot of permeance versus rejection, showing a trade-off between permeance and selectivity, also revealed that CMP membranes possess outstanding solvent permeance at similar selectivity (Supplementary Fig. 19).
In addition to rejection and permeance, another key parameter for evaluating the performance of OSN membranes is their stability. The outstanding permeance stability of CMP membranes was demonstrated in several typical organic solvents, including polar methanol, ethanol and non-polar hexane (Fig. 2e and Supplementary Fig. 20a), while their long-term permeance and rejection stability were manifested by continuous filtration of PPh-IX in ethanol for $100 \mathrm{~h}$ (Supplementary Fig. 20b). The high chemical and structural stability of CMP membranes was further indicated by testing filtration performance after soaking the membranes in different organic solvents for one week. As expected, the CMP membranes were highly stable in various organic solvents (Supplementary Fig. 21). Finally, the thermal stability was examined by thermogravimetric analysis 

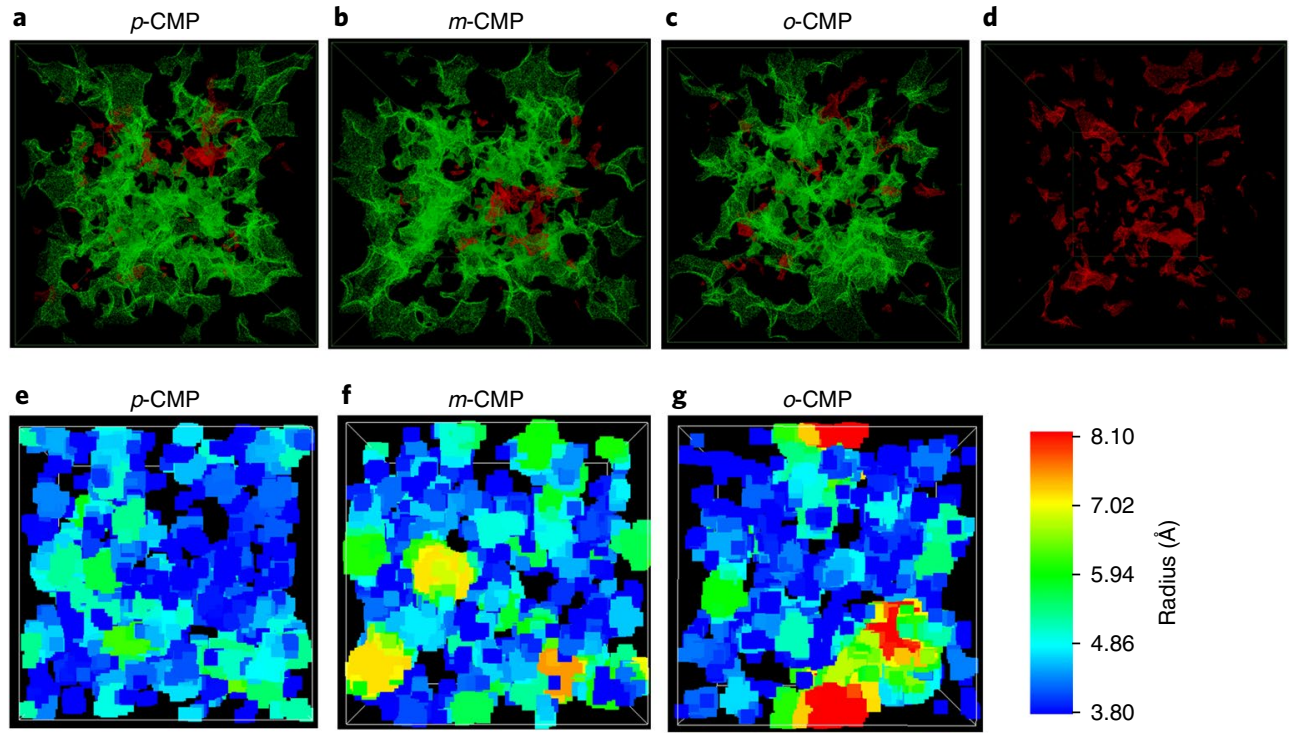

Fig. 3 | Structural analysis of amorphous polymer models. a-d, Interconnected (green) and disconnected (red) voids in $p$-CMP (a), $m$-CMP (b), o-CMP (c) and polyamide (d) with respect to a probe of $1.2 \AA$ radius, which represents the largest probe size that can diffuse across any of the rigid models. These images highlight the difference between all-rigid CMPs compared with conventional polyamide membranes. e-g, Voids coloured with respect to pore diameter for $p-C M P(\mathbf{e}), m-C M P(\mathbf{f})$ and $o-C M P(\mathbf{g})$.

(Supplementary Fig. 22), which showed high thermal stability of CMP membranes at $300^{\circ} \mathrm{C}$. Evidently, incorporation of the allconjugated skeleton rigidity into the polymers endows CMP membranes excellent mechanical, chemical and thermal robustness.

One intriguing characteristic of CMPs is their structural tunability. Theoretically, the pore surface chemistry of CMPs may be precisely controlled by pre-integrating different reactive moieties, which dramatically enriches the functionality of membranes. This concept was examined using hydroxyl-functionalized CMP; namely, $p$-CMP-OH with reactive $-\mathrm{OH}$ moieties on the pore surfaces. Alkyl moieties of different sizes were introduced by postgrafting (Supplementary Fig. 23), and the water contact angle of the membranes was found to increase from $86^{\circ}$ to more than $100^{\circ}$ (Supplementary Fig. 24), indicative of successful modification. $\mathrm{N}_{2}$ adsorption results revealed that the Brunauer-Emmett-Teller surface areas gradually decreased and the $\sim 2.2 \mathrm{~nm}$ pores in pristine $p$-CMP-OH membranes were blocked as the size of the grafted molecules increased (Supplementary Table 5 and Supplementary Fig. 25). As a result, the dye rejection significantly and progressively increased after modification with acetyl, benzoyl and adamantane formyl groups (Fig. 2f), accompanied by a dramatic permeance decrease from 15.4 to $\sim 1.51 \mathrm{~m}^{-2} \mathrm{~h}^{-1}$ bar $^{-1}$ (Supplementary Table 6), highlighting manipulation of the nanofiltration behaviour of CMP membranes at the molecular level.

Insight into the high OSN performances of CMP membranes. To understand the origin of high performance using CMPs as OSN membranes, molecular simulations were performed to construct realistic structural models. The amorphous polymer models were generated using Polymeric ${ }^{29}$-a simulated polymerization algorithm. Details of the simulations are given in the Supplementary Methods. Figure $3 \mathrm{a}-\mathrm{d}$ presents continuous and isolated voids with respect to a probe with a radius of $1.2 \AA$. All $p$-CMP, $m$-CMP and $o$ CMP membranes show high porosity and mostly continuous voids, confirming the hypothesis that using an all-conjugated rigid structure gives rise to membranes with enhanced microporosity and interconnectivity (Supplementary Figs. 26-28). High microporosity and interconnectivity are expected to cause continuous solvent flow, and this assumption was further verified by a simulation of continuous methanol flow through the $p$-CMP micropores (Supplementary Fig. 29). For comparison, the pore interconnectivities of polyamide and recently reported polyarylate and PIM with rigid monomers were also simulated using probes of different sizes (Supplementary Fig. 30). In sharp contrast, both polyamide and polyarylate membranes showed isolated voids, confirming their lower inner pore interconnectivity. Interestingly, PIM-EA-TB showed high porosity and good pore interconnectivity when a small $0.85 \AA$ probe was used, which indicated that rigid structure significantly increases porosity and interconnectivity. Figure $3 \mathrm{e}-\mathrm{g}$ further illustrates the voids, which are coloured with respect to the largest probe diameter that could be inserted. The pore-size distribution for each CMP polymer network was easily derived from the above simulation results, revealing that $p$-CMP, $m$-CMP and $o$-CMP have largest pore sizes of $0.65,0.75$ and $0.81 \mathrm{~nm}$, respectively (Supplementary Fig. 31). Generally, a larger pore size results in lower rejection; the simulated increased pore size was consistent with a decreased rejection performance of $p$-CMP, $m$-CMP and $o$-CMP (Fig. 2a). Of note, these simulated pore sizes were smaller than the real pore sizes tested by $\mathrm{N}_{2}$ adsorption and the diameter of the largest rejected markers, which suggests that the real pore size is underestimated in the simulated structure, probably due to neglect of the organic solvent in the simulation. Similar underestimation of the pore size was also observed in the polyarylate membrane, which was ascribed to the membrane being swollen in organic solvent ${ }^{21}$. Nevertheless, both simulations and experimental results clearly reveal the higher surface areas and porosity of the allrigid CMPs compared with traditional polyamide and polyarylate (Supplementary Table 2).

\section{Conclusions}

In summary, we developed a surface-initiated polymerization strategy for the fabrication of CMP membranes with high structural rigidity and microporosity, and show their great potential for application in OSN. We note that the CMP membranes exhibit very high permeance for a wide range of organic solvents with various polarities, and outperform all of the state-of-the-art polymer membranes (Supplementary Table 7). Additional advantages of CMP membranes include solute rejection with a distinct molecular weight cut-off of $560 \mathrm{~g} \mathrm{~mol}^{-1}$, high solvent resistance in various organic media, and 
tunable pores sizes and surfaces. Future scaled-up synthesis of CMP membranes might be achieved by direct surface-initiated polymerization on porous supports. This work may facilitate the development of next-generation polymer-based OSN membranes with permanent and abundant micropores via rational molecular design.

\section{Methods}

Synthesis of CMP membranes on functionalized silica wafers. To prepare the p-CMP membrane, 1-bromo-4-(trimethylsiloxy) benzene was first grafted onto a thin $\mathrm{SiO}_{2}$ layer via hydrolysis reaction. The as-grafted $\mathrm{SiO}_{2}$ substrate was then placed into a mixture of toluene and triethylamine $(1: 1 \mathrm{vol} / \mathrm{vol})$. The catalysts, tetrakis(triphenylphosphine)palladium $(0.012 \mathrm{mM})$ and $\mathrm{CuI}(0.012 \mathrm{mM})$ were added. Subsequently, the solution was stirred for $1 \mathrm{~h}$ at room temperature followed by the addition of 1,3,5-TEB $(0.1 \mathrm{mM})$ and 1,4-dibromobenzene $(0.15 \mathrm{mM})$. Afterwards, the reaction mixture was heated at $100^{\circ} \mathrm{C}$ for $72 \mathrm{~h}$. Finally, the resulting composite, $p$-CMP/silica, was washed with acetone, methanol, methylene chloride and diethyl ether, followed by drying under vacuum overnight. To prepare the $m$-CMP and $o$-CMP membranes, 1,3-dibromobenzene $(0.15 \mathrm{mM})$ or 1,2-dibromobenzene $(0.15 \mathrm{mM})$ was used as the monomer, respectively, with the rest of the procedure the same as for the $p$-CMP membrane.

CMP membrane transfer to an arbitrary porous support. The synthesized CMP membrane on $\mathrm{Si} / \mathrm{SiO}_{2}$ substrate was protected by a thin layer of poly(methyl methacrylate) (PMMA) by spin-coating PMMA solution (8 weight $\%$ in chlorobenzene) at 1,000 r.p.m. for 50 s. Then, the $\mathrm{SiO}_{2}$ layer was etched with diluted hydrofluoric acid (2\%) for $2 \mathrm{~h}$, resulting in a free-floating CMP/PMMA composite membrane. Following a previously reported transfer process for graphene membranes ${ }^{30}$, the CMP/PMMA composite membrane could be transferred to an arbitrary porous support and the PMMA protecting layer was removed using acetone.

Nanofiltration measurements. Nanofiltration experiments were performed in a laboratory-scale filtration unit, which included a dead-end filtration cell driven by $\mathrm{N}_{2}$ gas. The effective filtration diameter of membranes in this device was $0.5 \mathrm{~cm}$, supported by a porous stainless-steel disc. The selected CMP/PAN composite membrane was fixed at the bottom of the cell. The temperature of the feed solution in all experiments was maintained at $25^{\circ} \mathrm{C}$ using a water bath. Pure organic-solvent flux was obtained by measuring the mass of the solvent collected every 5 min under a steady state. The organic-solvent flux $(J)$ was calculated using the following equation:

$$
J=V /(A \times t)
$$

where $V$ is the volume of the organic solvent, $A$ is the effective membrane area and $t$ is the experimental time, respectively.

The concentrations of different dye solutions were determined using an ultraviolet visible spectrometer (Libra S32; Biochrom). The rejection $R$ (\%) was calculated using the equation:

$$
R=\left(1-C_{\mathrm{p}} / C_{\mathrm{f}}\right) \times 100 \%
$$

where $C_{\mathrm{p}}$ and $C_{\mathrm{f}}$ are the concentration of the permeate and feed solutions, respectively. All the experiments were carried out at least three times for each sample.

Generation of amorphous polymer models. Polymatic ${ }^{31,32}$-a simulated polymerization algorithm - was used to construct the amorphous polymer models, and all structures were described using the polymer consistent forcefield (PCFF). For CMP construction, three models were independently generated for each CMP polymer to ensure adequate sampling, and the properties were averaged across them. The bromines of dibromobenzene (DBB) and the hydrogens of the $\equiv$ $\mathrm{CH}$ group in 1,3,5-TEB were removed in the input molecular fragments, as they were not present in the final structure. Initially, a ratio of $3 \mathrm{DBB}$ molecules to 2 1,3,5-TEB molecules was inserted randomly in a periodic cubic cell, with cell dimensions of $70 \AA$, at low densities of $0.4 \mathrm{~g} \mathrm{~cm}^{-3}$. Polymerization steps were then performed sequentially. A new bond was added between 2 reactive atoms with less than $6 \AA$ distance, followed by energy minimization and molecular dynamics steps to maintain a relaxed structure throughout the simulated polymerization. The polymerization cycles were repeated until no pair meeting the bonding criteria was identified. The final polymerized structures were then annealed through a 21-step molecular dynamics equilibration as described previously ${ }^{32}$. The LAMMPS package ${ }^{33}$ was used for the polymerization algorithm and the 21-step relaxation procedure. We terminated any unreacted end groups after polymerization as reactants. For comparison, one model each was also generated for polyamide, polyarylate and PIM-EA-TB, respectively, following the same steps. Note that the repeat unit of PIM-EA-TB was defined according to the pervious method used for PIM-1 in ref. ${ }^{32}$

Data availability. The data that support the findings of this study are available within the paper and its Supplementary Information files, or from the corresponding author on reasonable request.
Received: 22 December 2017; Accepted: 30 May 2018; Published online: 23 July 2018

\section{References}

1. Marchetti, P., Jimenez-Solomon, M. F., Szekely, G. \& Livingston, A. G. Molecular separation with organic solvent nanofiltration: a critical review. Chem. Rev. 114, 10735-10806 (2014).

2. Cadotte, J. E., Petersen, R. J., Larson, R. E. \& Erickson, E. E. A new thin-film composite seawater reverse osmosis membrane. Desalination 32, 25-31 (1980).

3. Elimelech, M. \& Phillip, W. A. The future of seawater desalination: energy, technology, and the environment. Science 333, 712-717 (2011).

4. White, L. S., Wang, I. F. \& Minhas, B. S. Polyimide membrane for separation of solvents from lube oil. US patent US5 264, 166 (1993).

5. Linder, C., Nemas, M., Perry, M. \& Katraro, R. Silicone-derived solvent stable membranes. US patent US5 265, 734 (1993).

6. Dey, K. et al. Selective molecular separation by interfacially crystallized covalent organic framework thin films. J. Am. Chem. Soc. 139, 13083-13091 (2017)

7. Kandambeth, S. et al. Selective molecular sieving in self-standing porous covalent-organic-framework membranes. Adv. Mater. 29, 1603945 (2017)

8. Jimenez-Solomon, M. F., Bhole, Y. \& Livingston, A. G. High flux membranes for organic solvent nanofiltration (OSN)-interfacial polymerization with solvent activation. J. Membr. Sci. 423-424, 371-382 (2012).

9. Jimenez-Solomon, M. F., Bhole, Y. \& Livingston, A. G. High flux hydrophobic membranes for organic solvent nanofiltration (OSN) - interfacial polymerization, surface modification and solvent activation. J. Membr. Sci. 434, 193-203 (2013).

10. Karan, S., Jiang, Z. \& Livingston, A. G. Sub- $10 \mathrm{~nm}$ polyamide nanofilms with ultrafast solvent transport for molecular separation. Science 348, 1347-1351 (2015).

11. Freger, V. Outperforming nature's membranes. Science 348, $1317-1318$ (2015)

12. Guiver, M. D. \& Lee, Y. M. Polymer rigidity improves microporous membranes. Science 339, 284-285 (2013).

13. Carta, M. et al. An efficient polymer molecular sieve for membrane gas separations. Science 339, 303-307 (2013).

14. Park, H. B. et al. Polymers with cavities tuned for fast selective transport of small molecules and ions. Science 318, 254-258 (2007).

15. Du, N. et al. Polymer nanosieve membranes for $\mathrm{CO}_{2}$-capture applications. Nat. Mater. 10, 372-375 (2011).

16. Bezzu, C. G. et al. A spirobifluorene-based polymer of intrinsic microporosity with improved performance for gas separation. Adv. Mater. 24, 5930-5933 (2012)

17. Moneypenny, T. P. et al. Impact of shape persistence on the porosity of molecular cages. J. Am. Chem. Soc. 139, 3259-3264 (2017).

18. Alsbaiee, A. et al. Rapid removal of organic micropollutants from water by a porous $\beta$-cyclodextrin polymer. Nature 529, 190-194 (2016).

19. $\mathrm{Du}, \mathrm{Y}$. et al. Ionic covalent organic frameworks with spiroborate linkage. Angew. Chem. Int. Ed. 55, 1737-1741 (2016).

20. Gorgojo, P. et al. Ultrathin polymer films with intrinsic microporosity: anomalous solvent permeation and high flux membranes. Adv. Funct. Mater. 24, 4729-4737 (2014)

21. Jimenez-Solomon, M. F., Song, Q., Jelfs, K. E., Munoz-Ibanez, M. \& Livingston, A. G. Polymer nanofilms with enhanced microporosity by interfacial polymerization. Nat. Mater. 15, 760-767 (2016).

22. Jiang, J.-X. et al. Conjugated microporous poly(aryleneethynylene) networks. Angew. Chem. Int. Ed. 46, 8574-8578 (2007).

23. Xu, Y., Jin, S., Xu, H., Nagai, A. \& Jiang, D. Conjugated microporous polymers: design, synthesis and application. Chem. Soc. Rev. 42, 8012-8031 (2013)

24. Karan, S., Samitsu, S., Peng, X., Kurashima, K. \& Ichinose, I. Ultrafast viscous permeation of organic solvents through diamond-like carbon nanosheets. Science 335, 444-447 (2012)

25. Yang, Q. et al. Ultrathin graphene-based membrane with precise molecular sieving and ultrafast solvent permeation. Nat. Mater. 16, 1198-1202 (2017).

26. Mulder, M. Basic Principles of Membrane Technology (Kluwer Academic Publishers Group, Dondrecht, 1996).

27. Wijmans, J. G. \& Baker, R. W. The solution-diffusion model: a review. J. Membr. Sci. 107, 1 (1995).

28. Sorribas, S., Gorgojo, P., Téllez, C., Coronas, J. \& Livingston, A. G. High flux thin film nanocomposite membranes based on metal-organic frameworks for organic solvent nanofiltration. J. Am. Chem. Soc. 135, 15201-15208 (2013).

29. Abbott, L. \& Colina, C. Polymatic: A Simulated Polymerization Algorithm (nanoHUB, 2013); https://nanohub.org/resources/17278

30. Kim, K. S. et al. Large-scale pattern growth of graphene films for stretchable transparent electrodes. Nature 457, 706-710 (2009). 
31. Sun, H. Force field for computation of conformational energies, structures, and vibrational frequencies of aromatic polyesters. J. Comput. Chem. 15, 752-768 (1994).

32. Bayly, C. I., Cieplak, P., Cornell, W. \& Kollman, P. A. A well-behaved electrostatic potential based method using charge restraints for deriving atomic charges: the RESP model. J. Phys. Chem. 97, 10269-10280 (1993).

33. Plimpton, S. Fast parallel algorithms for short-range molecular dynamics. J. Comput. Phys. 117, 1-19 (1995).

\section{Acknowledgements}

This work was supported by the National Key Basic Research Program of China (2014CB931801 and 2016YFA0200700 to Z.T.), National Natural Science Foundation of China (51472054 to L.L.; 21475029,91427302 and 21721002 to Z.T.; and 11422215 and 11672079 to X.S.), Frontier Science Key Project of the Chinese Academy of Sciences (QYZDJ-SSW-SLH038 to Z.T.), Instrument Developing Project of the Chinese Academy of Sciences (YZ201311 to Z.T.), CAS-CSIRO Cooperative Research Program (GJHZ1503 to Z.T.) and Strategic Priority Research Program of the Chinese Academy of Sciences (XDA09040100 to Z.T.), K. C. Wong Education Foundation (to Z.T.), and Youth Innovation Promotion Association CAS (to L.L.). We thank H. Sun for discussion on PFQNM.

\section{Author contributions}

B.L., L.L. and Z.T. conceived the project, analysed the data and wrote the paper. H.W. and X.S. contributed to preparation of the manuscript. B.L. prepared the samples and performed the nanofiltration evaluation. X.H., Z.A.G., N.A.K., H.S. and A.M.K. characterized the samples. X.S., H.W. and B.S. performed the density functional theory calculations and simulations. All authors discussed the results and commented on the manuscript.

\section{Competing interests}

The authors declare no competing interests.

\section{Additional information}

Supplementary Information is available for this paper at https://doi.org/10.1038/ s41557-018-0093-9.

Reprints and permissions information is available at www.nature.com/reprints. Correspondence and requests for materials should be addressed to L.L. or Z.T.

Publisher's note: Springer Nature remains neutral with regard to jurisdictional claims in published maps and institutional affiliations. 